\title{
Angiomyolipoma of the Glabellar Region
}

\author{
Hyo Joong Kim, \\ Chan Min Chung, \\ Jae Yeon Park, \\ Sung Gyun Jung \\ Department of Plastic and Reconstructive \\ Surgery, National Medical Center, Seoul, \\ Korea
}

No potential conflict of interest relevant to this article was reported.

\begin{abstract}
Angiomyolipomas are hamartomatous lesions consisting of smooth muscle bundles, thickwalled blood vessels, and mature adipocytes. They are usually found in the kidneys of tuberous sclerosis patients and more rarely in other organs, such as the liver, the oral cavity, the sinonasal tract, the heart, the large intestines, the lungs, and the skin. Cutaneous angiomyolipoma has shown to be very rare and generally occurs at the ends of the digits, the elbows, the ears, and the nose. Herein, we report the first documented case of angiomyolipoma of the glabellar region.
\end{abstract}

Keywords: Angiomyolipoma / Perivascular epithelioid cell neoplasms / Angiolipoleiomyoma / Tuberous sclerosis

\section{INTRODUCTION}

Angiomyolipomas are usually found in the kidneys of tuberous sclerosis patients. They are also sometimes seen in patients with polycystic kidney disease, with neurofibromatosis type 1 , or with von Hippel-Lindau syndrome [1]. Less frequently, they have been found to occur in organs such as the liver, the oral cavity, the nasal cavity, the heart, the large intestines, and the lungs. Angiomyolipomas of the skin are extremely rare. Cutaneous angiomyolipomas have mostly been discovered at the terminal ends of the body, such as the distal phalanges of the finger and the foot, the elbows, the ears, and the nose. Cutaneous angiomyolipomas usually measure 1-4 cm. Regarding their putative etiology, Obata et al. [2] suggested that a neglected lesion in distal body parts becomes degenerated upon trauma and is replaced by adipose tissue. The case described in this study is the first reported diagnosis of an angiomyolipoma of the glabella. In many respects, cutaneous angiomyolipoma shares similar histological features

Correspondence: Chan Min Chung

Department of Plastic and Reconstructive Surgery, National Medical Center, 245

Euljiro, Jung-gu, Seoul 04564, Korea

E-mail: nmcps.doctor@gmail.com

Received March 6, 2017 / Revised August 9, 2017 / Accepted August 9, 2017 to the renal angiomyolipomas found in tuberous sclerosis patients. However, some differences allow us to distinguish between cutaneous angiomyolipoma and renal angiomyolipoma as separate entities. First, cutaneous angiomyolipomas have not been shown to be associated with tuberous sclerosis. Accordingly, the typical cutaneous lesions of tuberous sclerosis patients, such as hypopigmented macules, shagreen patches, and angiofibromas, were absent in our patient. Second, most renal angiomyolipomas stain positive for human melanoma black antibody (HMB)-45 (95\% muscular), while most cutaneous angiomyolipomas do not [3]. Finally, renal angiomyolipoma has a female predominance, while cutaneous angiomyolipoma has a male predominance.

In this case report, we report a male patient with a glabellar mass that increased in size over the course of 3 years. He was otherwise in good health condition without any trauma, surgical or medical history. After surgical excision and pathological study, we finally arrived at a final diagnosis: cutaneous angiomyolipoma at the glabellar region. As far as to our knowledge we report first ever case of a glabellar angiomyolipoma. 


\section{CASE REPORT}

A 60-year-old male patient was admitted to our hospital for a possible cancerous mass on the glabellar region that had gradually grown over 3 years to a size of a walnut. The patient, other than having a 20-year work history as a pelagic fisherman, was absent of any notable medical history or traumatic injuries. Upon physical examination, the mass was seen to be without flare or discharge and soft in consistency without any tenderness, and measured $3.0 \times 2.5 \mathrm{~cm}$ in size. It had grown to occupy a substantial area between the nasions of the glabella (Fig. 1).

The patient did not present with other abnormalities, nor systemic signs associated with tuberous sclerosis simplex, such as hypopigmented macules, facial angiofibromas, or shagreen patches. The patient had no notable family history. We surgically excised the mass with the patient under general anesthesia. The excised tumor mass, which measured $2.3 \times 1.7 \mathrm{~cm}$, was oval, soft, and well-encapsulated (Fig. 2). We observed many thick-walled blood vessels and mature adipocytes on hematoxylin and eosin staining (Fig. 3).

Many thck-walled blood vessels and mature adipocyte are observed. The muscle tissue was stained using Masson trichrome, and the walls of the blood vessels were stained using friend leuke-

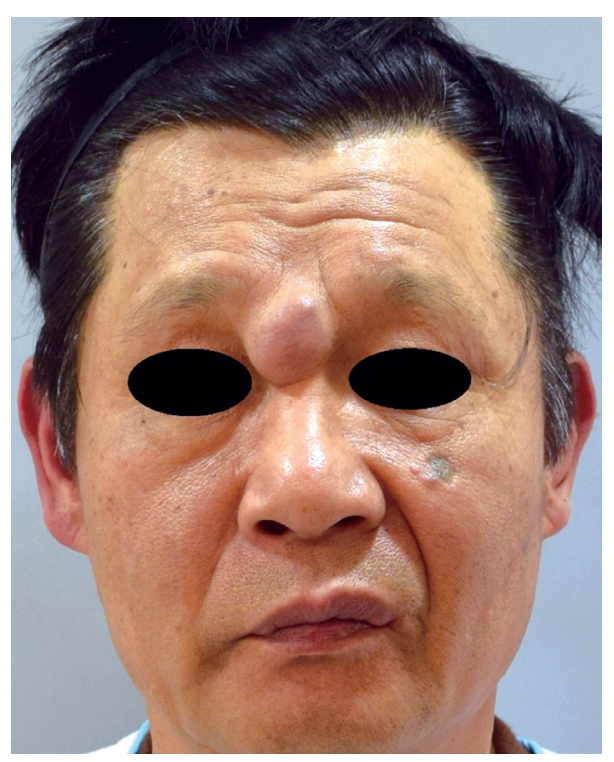

Fig. 1. A 60 -year-old male patient with $3.0 \times 2.4 \mathrm{~cm}$ mass at the glabellar region.

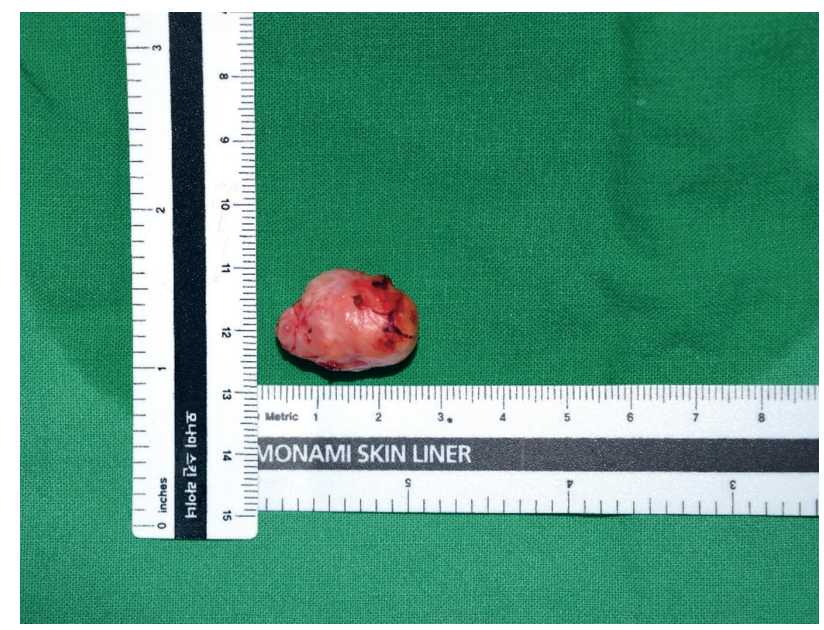

Fig. 2. Gross view. The excised tumor was a well encapsulated, $2.3 \times 1.7 \mathrm{~cm}$, ovoid, soft mass.

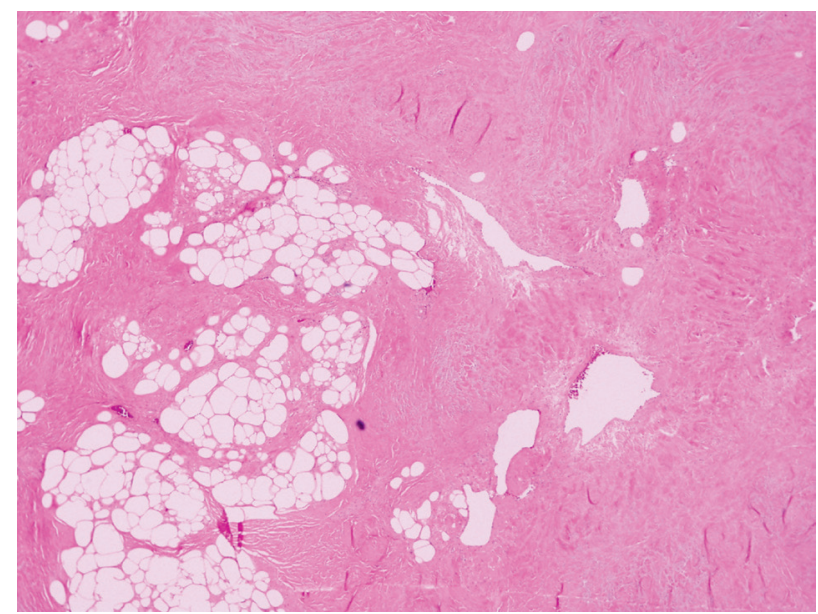

Fig. 3. Many thick-walled blood vessels and mature adipocyte are observed (H\&E, $\times 40)$.

mia integration 1 transcription factor (FLI-1) (Fig. 4). There is negative staining result using HMB-45 (Fig. 5). The muscle tissue was stained red using Masson trichrome, and the walls of the blood vessels were stained using FLI-1. No positivity of HMB-45 stain. The sutures were removed at the end of the first postoperative week, and at the 15-month follow-up, we observed no recurrence or side effects (Fig. 6).

\section{DISCUSSION}

Angiomyolipoma (AML), also referred to as angiolipoleiomyoma 

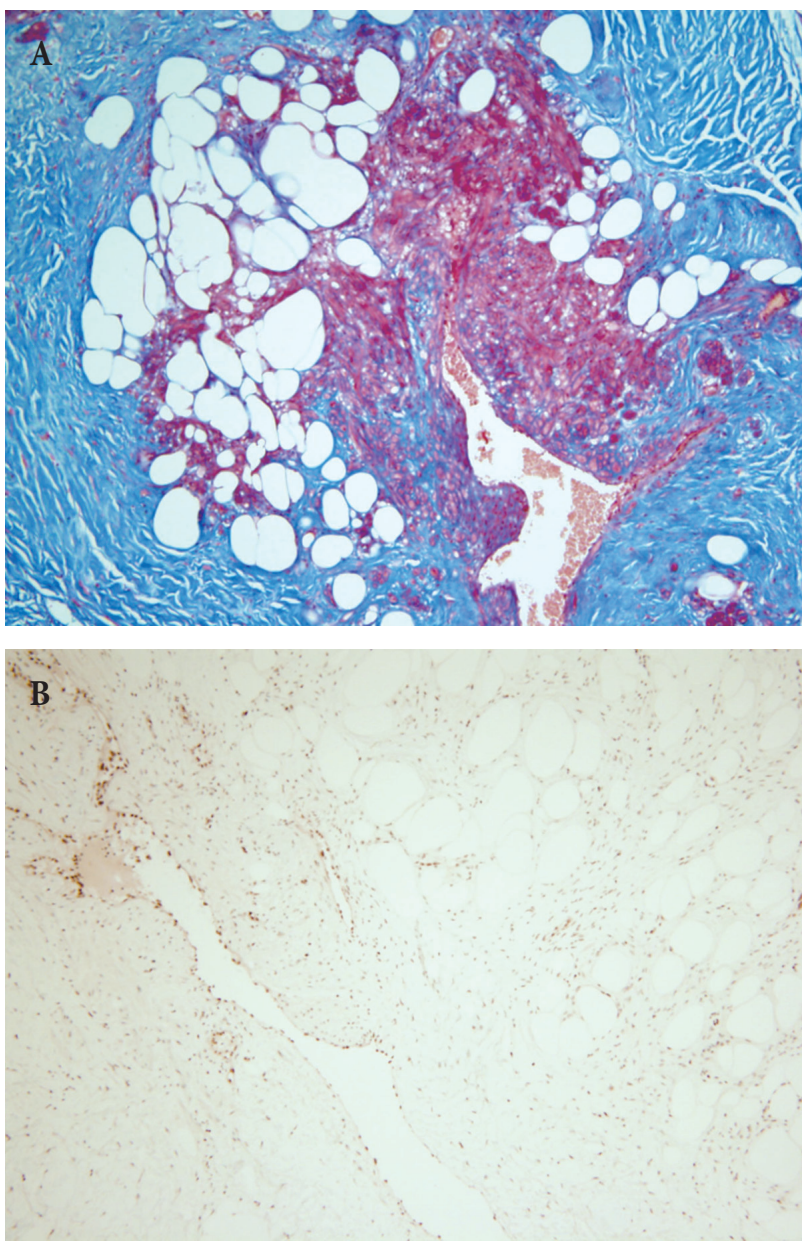

Fig. 4. (A) Masson trichrome stain (×100), and (B) FLI-1 (blood vessel specific) stain $(\times 100)$. The muscle tissue was stained red using Masson trichrome, and the walls of the blood vessels were stained using FLI-1. FLI-1, friend leukemia integration 1 transcription factor.

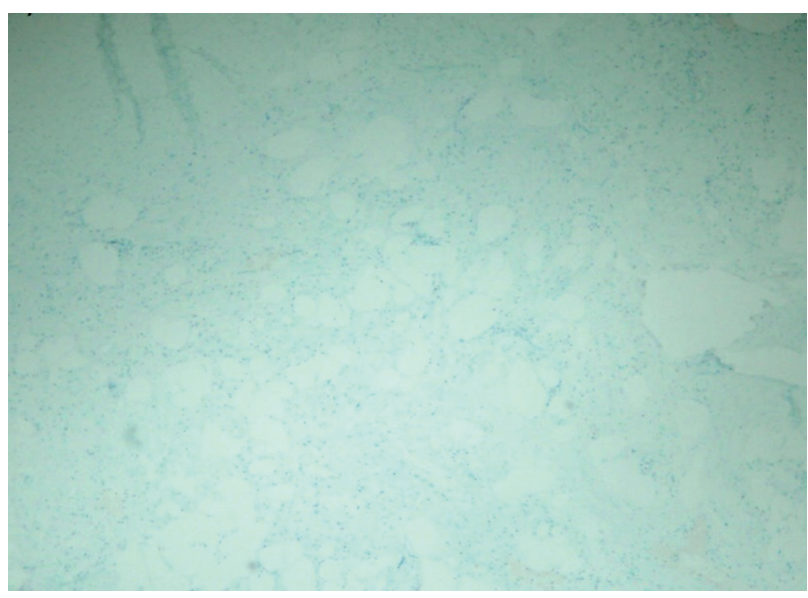

Fig. 5. HMB-45 stain $(\times 40)$. No positivity of HMB-45 stain. HMB, human melanoma black antibody.

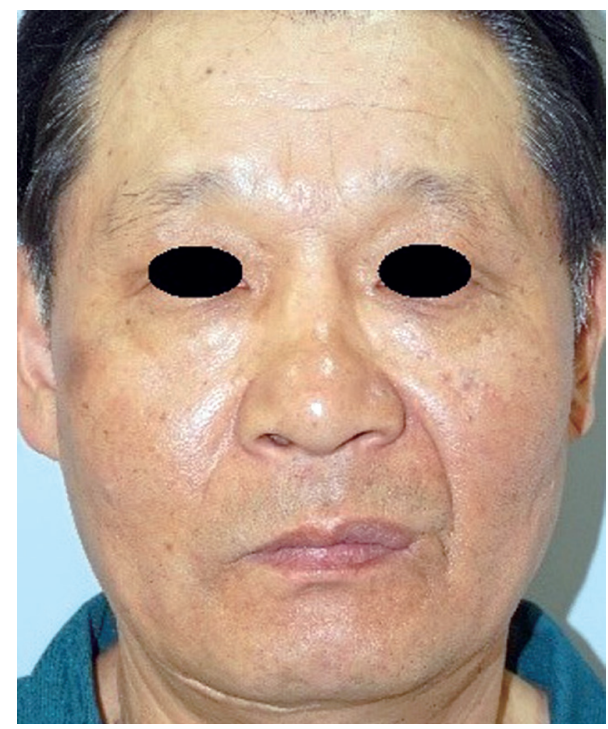

Fig. 6. Follow-up photograph at postoperative 15 months showing no signs of recurrence.

(ALLM) [4], is classified as a hamartoma that belongs to a family of tumors arising from perivascular epithelioid cells (PEComas) [5]. Hamartoma is a focal malformation that resembles a benign neoplasm in that it is composed of tissue elements normally found in that tissue but grows in a disorderly fashion [3]. AML is defined histologically as a benign pleomorphic mesenchymal tumor consisting of three histologic components: mature adipocytes, interlacing fascicles of smooth muscle cells, and abnormal tortuous convoluted thick-walled blood vessels without an elastic layer [3,69]. This classical triad is necessary for a definitive diagnosis [7]. Depending on the frequency of occurring site, angiomyolipoma can be further categorized into renal AML and extrarenal AMLmajority of AML's are renal AML ( $99.7 \%$ of all AMLs, $1 \%$ of all renal tumors [9]; $0.1 \%-0.3 \%$ incidence $[10,11]$ ) while very few comprise the extrarenal AML (0.3\% of all AMLs) [12]. The overall incidence of angiomyolipoma is estimated to be $0.3 \%$ in the general population [13]. Because most of AMLs are renal AML (99.7\%) and extrarenal AML $(0.1 \%-0.3 \%)$ are extremely rare, it is obvious that the incidence of renal AML is almost similar to the overall incidence of AML per se. The extrarenal sites include the liver, the retoperitoneal area, nasal cavity, oral cavity, vagina, fallopian tubes, spinal cord, spermatic cord, penis, heart, colon, lung, bone and the skin $[9,14,15]$. 
AML can be found either in conjunction with tuberous sclerosis complex (TSC) (20\%) or in a sporadic form (=isolated form) (80\%) [13]. Once identified, renal AML is pathognomonic finding of TSC [12]. Renal AML can be identified in association not only with TSC (although most commonly) but also with other kidney diseases such as adult polycystic kidney disease, neurofibromatosis type 1, or von Hippel Lindau disease [4]. Among those who are diagnosed with TSC, $80 \%$ will have renal lesions [13]. Roughly one third (33\%) of TSC patients will have renal AML [8]. Inversely, approximately $40 \%$ of patients with AML and TSC show stigmas or signs of adenoma sebaceum, ash-leaf macule, shagreen patches, or café au-lait macules [4]. Other signs of typical TSC include epilepsy (convulsive crises), mental retardation, oligophrenia, and renal AML $[9,15]$. It is important to emphasize that renal lesions are as common as $60 \%-80 \%$ [16] of all TSC patients and this even higher involvement can be explained by the fact that renal AML and renal cyst are the two most commonly occurring renal lesions in TSC [17].

Although TSC is an autosomal dominant hereditary disease that involves chromosome 9 or chromosome 16 [15] and mutations in tumor suppressor gene TSC1 (encodes protein hamartin) or TSC2 (encodes protein tuberin) genes [17,18], sporadic form that might arise from spontaneous mutations, have also been reported [15]. TSC is a multisystem disease with autosomal dominant inheritance, variable expressivity, and high penetrance that can be characterized with seven different hamartomas (cortical tubers, subependymal nodule, retinal phakoma, facial angiofibromas, ungual firbromas, fibrous forehead plaque, and multiple renal angiomyopliomas) [12]. A definitive diagnosis can be made when more than two are found [12].

The renal AMLs are usually found incidentally because of their hidden location and often are asymptomatic. They however have a tendency to grow or become invasive leading to possible complications such as spontaneous retroperitoneal bleeding [17] that could in turn bring about life threatening conditions such as hypovolemic shock (occurring in one third of retroperitoneal hemorrhage) $[9,10]$. This complication rate is commensurate with the size of the tumor, histologic grade of the tumor (proportional to the extent of neovascularization), and the presence of tuberous sclerosis [11]. Tumor size greater than $4 \mathrm{~cm}$ warrants close monitoring with imaging modalities (i.e., MRI/MR angiography or Doppler ultrasound) [17] and prompt intervention [9,10]. Another possible complication that is regarded as a medical emergency is called the Wunderlich syndrome that presents with spontaneous perinephric space bleeding without any trauma.

The extrarenal AML has several synonyms that include mucocutaneous AML (MCAML) (proposed by Watanabe and Suzuki, 1999) [18], Cutaneous AML, and Cutaneous angiolipoleiomyoma (CALLM) [7,14]. Cutaneous AMLs are usually painless solitary lesions located in the acral area [4].

Due to its modicum of knowledge in the literature, only very little is known about the characteristics of cutaneous angiomyolipoma. These characteristics dictates its entitiy perhaps as a separate disease.

Albeit sharing similar pathological findings, renal AML and the extrarenal AML can be regarded as disparate disease entities since they behave totally different from each other in terms of hisotology, clinical behavior and demographics. These different characteristics can be summarized as follows. First of all, renal AML has small to medium sized with thickened blood vessels with epithelioid components, while extrarenal AML has large blood vessels often without epithelioid components [4]. In addition, renal AML shows signs of TSC up to $30 \%$ while in the extrarenal AML, except for the liver, patients are not afflicted with TSC and have excellent prognosis $[8,14]$. Moreover, in contrast to the renal AML which may be invasive and may recur [7], and display HMB-45 positivity in 95\% of the patients [14,15], extrarenal AML has no recurrences and lack positivity to HMB-45 [16]. Demographically, sex and age separate these two entities: the renal AML has a female predominance, while the extrarenal AML has a male (male:female=12:2) predominance $[4,12,14,19]$. In respect to age, renal AML presents in the younger age bracket than its counterpart extrarenal AML (5th or 6th decades of life) [12,19].

Among the extrarenal AMLs, the liver is by far the most common site $[7,12]$ followed by the retroperitoneal region [20]. AMLs' occurring as hepatic lesions may present in association with TSC and may behave as an exception from the extrarenal AMLs [12]. By the same token, the retroperitoneal AML can also be regarded 
as an exception to this tendency since malignant cases were reported although most of them exhibit in benign forms [20].

Our report illustrates that even cancerous masses found in regions, not usually associated with cutaneous angiomyolipomas, must undergo a differential diagnosis that excludes cutaneous angiomyolipomas, and presents previously unknown diagnostic characteristics of extrarenal angiomyolipomas.

\section{PATIENT CONSENT}

The patient provided written informed consent for the publication and the use of their images.

\section{REFERENCES}

1. Argenyi ZB, Piette WW, Goeken JA. Cutaneous angiomyolipoma: a light-microscopic, immunohistochemical, and electron-microscopic study. Am J Dermatopathol 1991;13:497-502.

2. Obata C, Murakami Y, Furue M, Kiryu H. Cutaneous angiomyolipoma. Dermatology 2001;203:268-70.

3. Pontes HA, Pontes FS, Cruz e Silva BT, Martel LP, Carneiro JT, Silva BS, et al. Angiomyolipomatous hamartoma of the upper lip: a rare case in an 8-month-old child and differential diagnosis. J Craniomaxillofac Surg 2011;39:102-6.

4. Shim HS, Kim DH, Kwon H, Jung SN. Cutaneous angiomyolipoma in the forehead. J Craniofac Surg 2014;25:1120-2.

5. Chen GX, Wang MH, Hu CL, Tang GC, Han FG. Angiomyolipoma of the rib: a rare case report. Mol Clin Oncol 2016;4:126-8.

6. Bauer V, Aleric Z, Bujas T. Huge angiomyolipoma of the tongue. Otolaryngol Head Neck Surg 2012;146:512-3.

7. Ammanagi AS, Dombale VD, Shindholimath VV. Cutaneous angiomyolipoma. Indian Dermatol Online J 2013;4:255-6.

8. Ide F, Shimoyama T, Horie N. Angiomyolipomatous hamartoma of the tongue. Oral Surg Oral Med Oral Pathol Oral Radiol Endod 1998; 85:581-4.
9. Coscaron Blanco E, Gomez Gonzalez JL, Blanco Perez P, Canizo Alvarez A, Benito Gonzalez F, Flores Corral T. Cervicothoracic angiomyolipoma: an unusual tumor located at a site difficult to reach for surgery. Acta Otorrinolaringol Esp 2004;55:148-51.

10. Maclean DF, Sultana R, Radwan R, McKnight L, Khastgir J. Is the follow-up of small renal angiomyolipomas a necessary precaution? Clin Radiol 2014;69:822-6.

11. Ploumidis A, Katafigiotis I, Thanou M, Bodozoglou N, Athanasiou L, Ploumidis A. Spontaneous retroperitoneal hemorrhage (Wunderlich Syndrome) due to large upper pole renal angiomyolipoma: does robotic-assisted laparoscopic partial nephrectomy have a role in primary treatment? Case Rep Urol 2013;2013:498694.

12. Gatalica Z, Lowry LD, Petersen RO. Angiomyolipoma of the nasal cavity: case report and review of the literature. Head Neck 1994;16:278-81.

13. Iwata AJ, Friedmann DR, Kaplan J, Wang BY, Lebowitz RA. A man with recurrent right-sided epistaxis: angiomyolipoma (AML) of the nasal cavity. JAMA Otolaryngol Head Neck Surg 2013;139:1067-8.

14. Shin JU, Lee KY, Roh MR. A case of a cutaneous angiomyolipoma. Ann Dermatol 2009;21:217-20.

15. Foschini MP, Corti B, DaCol M, Cenzi M, Zanella F, Barbazza R. Angiomyolipoma of the parotid gland: a case report. Oral Surg Oral Med Oral Pathol Oral Radiol Endod 1999;87:738-41.

16. Rosado P, Villalain L, De Vicente JC, Vivanco B, Torre A. Angiomyolipoma of the parotid gland: report of a case and review of the literature. J Oral Maxillofac Surg 2010;68:2609-12.

17. Tsai JD, Wei CC, Chen SM, Lue KH, Sheu JN. Association between the growth rate of renal cysts/angiomyolipomas and age in the patients with tuberous sclerosis complex. Int Urol Nephrol 2014;46:168590

18. Ieremia E, Robson A. Cutaneous PEComa: a rare entity to consider in an unusual site. Am J Dermatopathol 2014;36:e198-201.

19. Carrau D, Kahwash S, Pearson G. Cutaneous angiomyolipoma masquerading as a neurofibroma in a child with neurofibromatosis type 1 : a case report. Pediatr Dermatol 2015;32:423-4.

20. Vilcea ID, Victor R, Mirea CS, Simionescu CE, Mogoanta SS, Moraru E, et al. Extrarenal retroperitoneal angiomyolipoma with unusual evolution. Rom J Morphol Embryol 2015;56:263-6. 